\title{
A Broken Heart: A Rare Complication of Hyponatremia
}

\author{
Adam Purdy ${ }^{\mathrm{a}, \mathrm{b}}$, Bibai Ren ${ }^{\mathrm{a}}$
}

\begin{abstract}
We present a case of a 47 -year-old female with a history of anxiety, depression, and alcohol dependence with history of alcohol withdrawal seizures, who presented after a witnessed seizure episode. She admitted to drinking only copious amounts of black tea 1 week prior to presentation in an attempt to quit alcohol. She was subsequently found to be severely hyponatremic with a serum sodium level of 112 $\mathrm{mEq} / \mathrm{L}$ and was admitted to the intensive care unit. Approximately 48 $\mathrm{h}$ after admission, she experienced sudden-onset of dizziness, diaphoresis, and nausea, with a heart rate of 42 beats/min and blood pressure of 70/40 $\mathrm{mm} \mathrm{Hg}$. STAT EKG revealed new T-wave inversions in the lateral and precordial leads and troponin was elevated to $5.99 \mathrm{ng} /$ $\mathrm{mL}$. Treatment was initiated for NSTEMI with continuous heparin infusion, aspirin, clopidogrel, carvedilol, and lisinopril. Transthoracic echocardiogram revealed apical and mid ventricular dyskinesia with hyperkinesis of the basal walls, with an estimated left ventricular ejection fraction of $35 \%$. Cardiac catheterization revealed no significant coronary disease with ejection fraction $30-35 \%$ and similar areas of hypo and hyperkinesis, classic for apical ballooning syndrome. This case not only illustrates the rare phenomenon of hyponatremiainduced Takotsubo cardiomyopathy, but is one of the only incidents caused by primary polydipsia.
\end{abstract}

Keywords: Takotsubo cardiomyopathy; Primary polydipsia; Hyponatremia; Stress cardiomyopathy; Stress-induced cardiomyopathy; Apical ballooning syndrome; Broken heart syndrome

\section{Introduction}

Stress cardiomyopathy, also known as Takotsubo cardiomyopathy, apical ballooning syndrome, or broken heart syndrome, is a transient decrease in left ventricular function, mimicking acute coronary syndrome (ACS) in symptoms, electrocardiogram, and laboratory findings, but lacks significant coronary artery occlusion or plaque rupture on angiography. Though its pathogenesis is not well understood, proposed causes include

Manuscript submitted March 4, 2018, accepted April 19, 2018

${ }^{a}$ Department of Medicine, Overlook Medical Center, Summit, NJ 07901, USA ${ }^{b}$ Corresponding Author: Adam Purdy, Department of Medicine, Overlook Medical Center, 99 Beauvoir Avenue, Summit, NJ 07901, USA.

Email: adam.g.purdy@gmail.com

doi: https://doi.org/10.14740/jmc3050e catecholamine excess, coronary artery spasm, and rarely, hyponatremia. Stress cardiomyopathy should be a strong consideration in patients who present with hyponatremia caused by primary polydipsia and develop symptoms of ACS without significant risk factors.

\section{Case Report}

A 47-year-old female with past medical history of alcohol abuse, depression, and anxiety presented to the hospital after witnessed tonic-clonic seizures at home. She had no other significant medical history, such as diabetes, hypertension, hyperlipidemia, history of tobacco or drug use, or family history of cardiac disease. She did have a history of alcohol withdrawal seizures, occurring 2 years prior to presentation. She drank 6 - 10 (12 oz.) beers per day for the past 10 years with the last drink being 1 week prior to presentation. In an attempt to detoxify, she drank up to 12 cups of black tea per day during this time with minimal food intake. At the time of initial evaluation, she was asymptomatic and at baseline. Home medications included daily aripiprazole, fluoxetine, mirtazapine, and gabapentin.

On initial evaluation, she was found to be tachycardic with blood pressure of 144/88 mm Hg. She was resting comfortably in no acute distress. Her tongue was noted to be black in color with moist mucous membranes. Her cardiac exam, besides elevated rate, had no appreciable arrhythmia, murmurs, rubs, or gallops. Respiratory exam revealed clear lungs and normal breath sounds. Her complete blood count was within normal range and her metabolic panel was pertinent for a sodium level of $112 \mathrm{mmol} / \mathrm{L}$ ( $139 \mathrm{mmol} / \mathrm{L}, 18$ months prior), with a serum osmolality of $259 \mathrm{mOsm} / \mathrm{kg}$, AST of $49 \mathrm{U} / \mathrm{L}$, and ALT of $28 \mathrm{U} / \mathrm{L}$. Ethanol level was $<10 \mathrm{mg} / \mathrm{dL}$. CT of head was unremarkable and chest X-ray was clear. EKG revealed sinus tachycardia with mild diffuse ST elevation, likely secondary to early repolarization changes (Fig. 1a). Pertinent urine studies revealed a urine osmolality of $68 \mathrm{mOsm} / \mathrm{kg}$ and urine electrolytes were significant for sodium of $18 \mathrm{mmol} / \mathrm{L}$.

She was admitted to the intensive care unit for alcohol withdrawal seizures and severe hyponatremia secondary to primary polydipsia. Her hyponatremia was corrected appropriately with intravenous fluids and alcohol withdrawal was treated with a benzodiazepine taper with no further seizures.

Approximately $48 \mathrm{~h}$ after admission, she experienced sudden-onset of dizziness, diaphoresis, nausea, and lethargy. At that time, she was bradycardic to $42 \mathrm{bpm}$ and hypotensive to $70 / 40 \mathrm{~mm} \mathrm{Hg}$. This episode lasted approximately $3 \mathrm{~min}$ and resolved without intervention. She had no chest pain, palpita- 


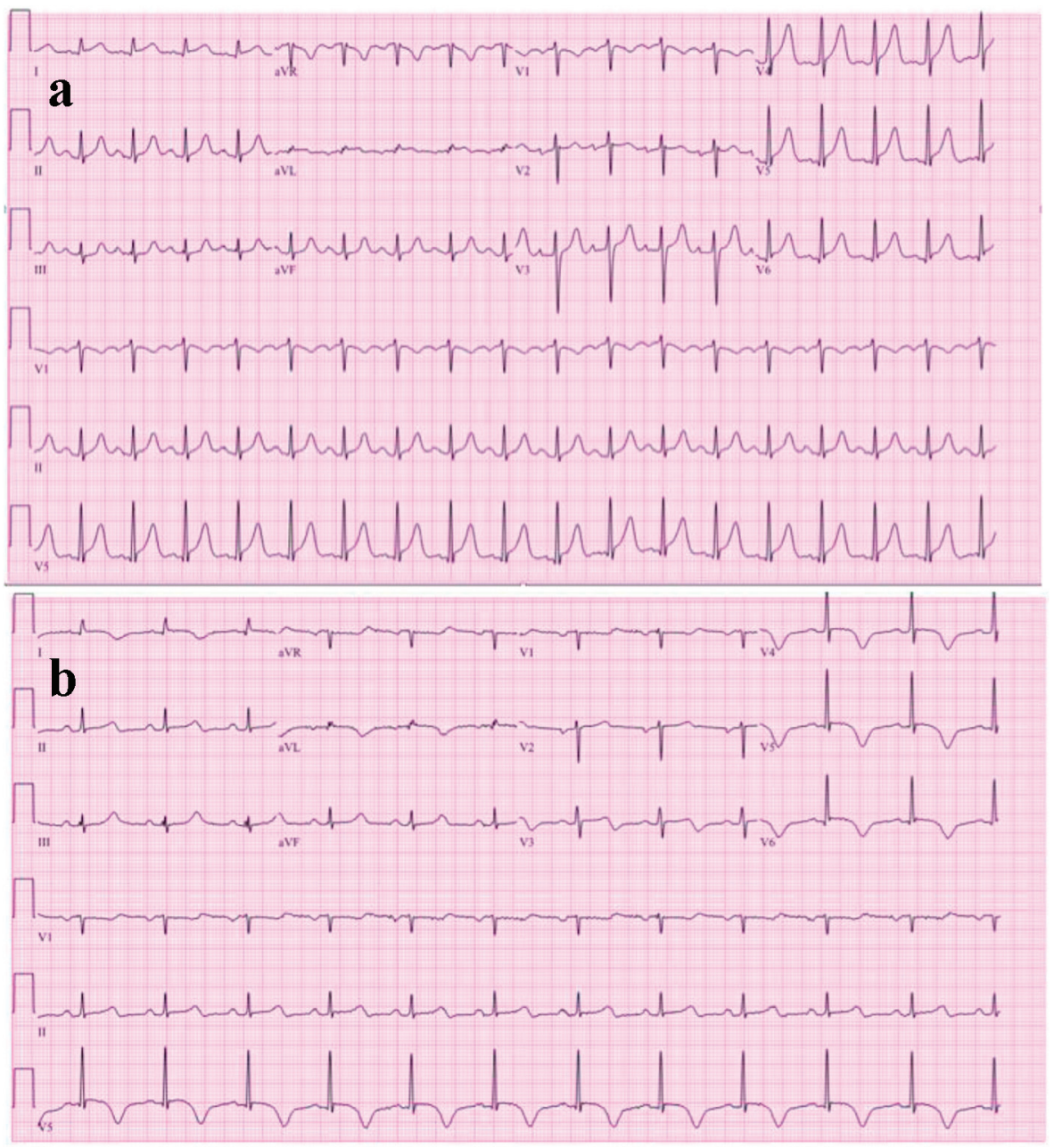

Figure 1. (a) Initial electrocardiogram showing sinus tachyarrhythmia with mild ST-segment elevations likely early repolarization and no T-wave inversions. (b) Repeat EKG, showing new T-wave inversions in I, aVL, and V3-V6.

tions, or shortness of breath. A STAT EKG at the time of the event showed new T-wave inversions in leads I, aVL and V3V6 (Fig. 1b). Serum troponin drawn at the time of the episode was elevated at $5.99 \mathrm{ng} / \mathrm{mL}$. Standard treatment for non-ST elevation ACS was initiated with continuous heparin infusion, aspirin, clopidogrel, carvedilol, and lisinopril. Lipid profile and glycosylated hemoglobin were within normal limits. Echocardiogram revealed apical and mid ventricular dyskinesia with hyperkinesis of the basal walls, with an estimated left ventricular ejection fraction of 35\% (Fig. 2). Cardiac catheterization revealed mild coronary artery disease, a left ventricular ejection fraction of 30-35\%, hyperkinesis of the basal inferior walls, as well as apical and mid anterolateral, and mid inferior dyskinesis, classic for stress cardiomyopathy (Fig. 3).

\section{Discussion}

Stress cardiomyopathy, also known as Takotsubo cardiomyopathy or apical ballooning syndrome is a transient decrease in left ventricular function, acting similarly to ACS, reported in $1-2 \%$ of patients presenting with ACS [1]. It is a diagnosis made after excluding coronary artery disease and plaque rupture and is characterized by depressed mid and apical segments of the left ventricle, and hyperkinesis of the basal walls. It is most common in postmenopausal women and in an International Takotsubo Registry study, 55\% of patients diagnosed with Takotsubos had an acute, former, or chronic psychiatric or neurologic disorder [2]. The exact mechanism for this syndrome is unclear, but hypotheses include catecholamine excess, coronary artery spasm, and microvascular dysfunction [3].

The clinical presentation is similar to that of ACS, most commonly acute substernal chest pain. Others may present with dyspnea, syncope, tachyarrhythmias, bradyarrhythmias, diaphoresis, new murmur, or even cardiogenic shock. This phenomenon is more often seen in women and predominantly seen in older adults. Similarly to ACS, EKG most commonly shows ST segment elevations, but may show ST depressions, QT prolongation, abnormal Q waves, or T-wave inversions $[3,4]$. Cardiac biomarkers, including troponin and CKMB are usually elevated. Diagnosis is made based on transient left 

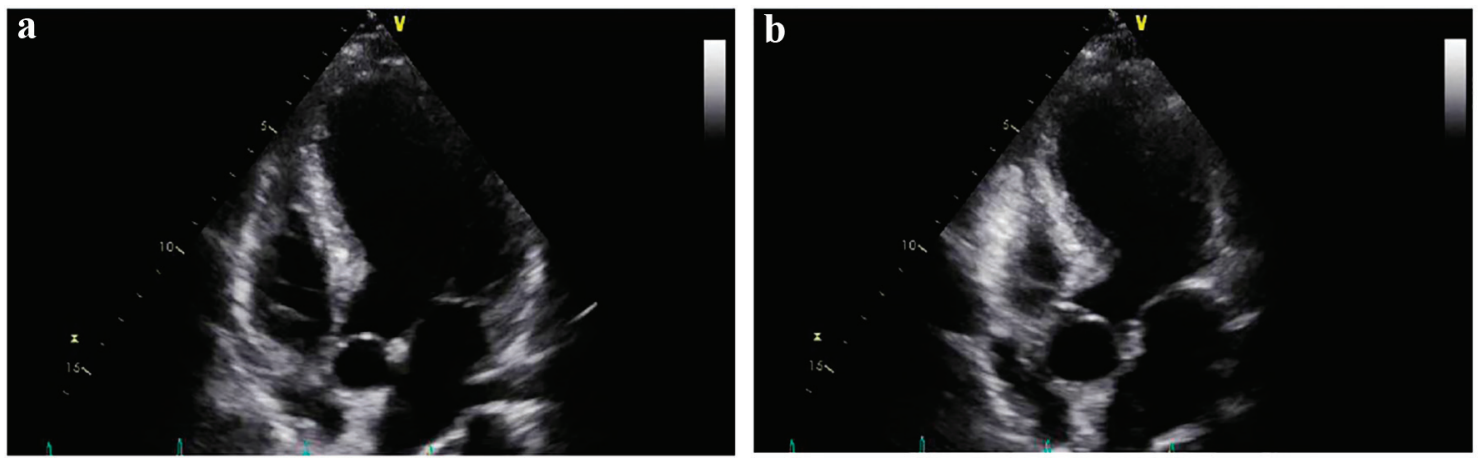

Figure 2. (a) Four chamber view on transthoracic echocardiogram during diastole. (b) Four chamber view on transthoracic echocardiogram showing dilatation of the apical portion of the left ventricle during systole.

ventricular dysfunction, elevated cardiac biomarkers, EKG changes, and exclusion of obstructive coronary disease and plaque rupture. There are no evidence-based guidelines for treatment of Takotsubo, but standard heart failure medications are usually started, including a beta blocker, ACE inhibitor, aspirin, and statin $[5,6]$.

In the vast majority of cases, Takotsubo cardiomyopathy is triggered by an emotionally or physically stressful event that usually precedes the onset of symptoms by minutes to hours. Emotional examples are not limited to extreme grief, fear, anger, relationship conflicts, and financial hardships, which were linked to $28 \%$ of 1,759 cases in the International Takotsubo Registry Study [2, 4]. Physical stressors may include acute asthma exacerbation, major surgery, chemotherapy, or stroke, and were linked to $36 \%$ of the cases. Eight percent of cases included both emotional and physical triggers, while $29 \%$ had no evident trigger [2]. It has rarely been documented that a severe electrolyte imbalance can play a role in the development of this stress-induced cardiomyopathy.

Seizures have been shown to play a role in Takotsubo cardiomyopathy [7]. Our patient, however, developed cardiomyopathy $48 \mathrm{~h}$ after witnessed seizure activity. She was not known to have an underlying seizure disorder, as hyponatremia seemed to be the clear inciting event, with her last alcoholic

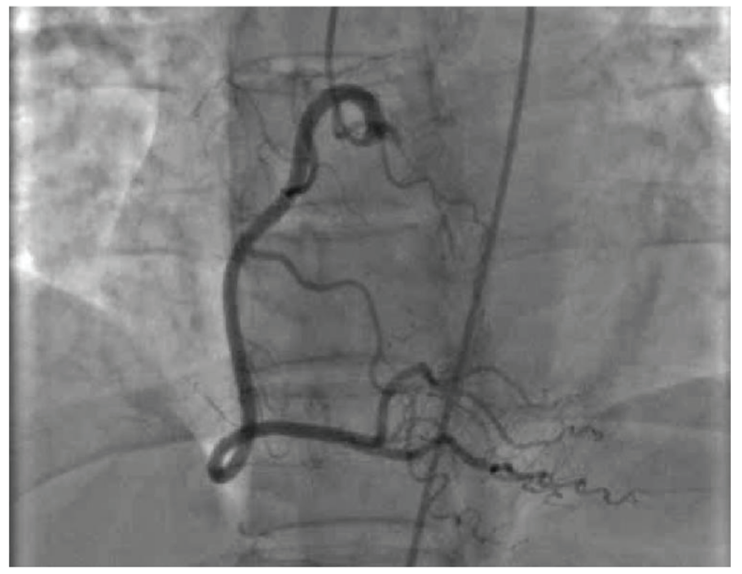

beverage being 1 week prior. She also did not report any acute illness, recent surgery, or emotional stressor, confirmed by family members, leaving hyponatremia as the sole cause of her cardiomyopathy. After extensive literature search, just a handful of case reports (five) have documented hyponatremia as a cause of Takotsubo cardiomyopathy without the presence of adrenal insufficiency and hypothyroidism. Three of these cases were associated with inappropriate ADH secretion, one with diuretic use, and one of an unknown etiology causing hyponatremia [8]. Our patient, however, exemplifies a case of primary polydipsia causing hyponatremia (from her excessive tea intake), an entity not previously reported. Although the cause of hyponatremia-induced catecholamine surge remains unknown, excess catecholamines, particularly norepinephrine and dopamine, seem to play an important role in the development of stress-induced cardiomyopathy [9]. Despite the correction of our patient's metabolic derangement without neurological deficits, she was left with the sequelae of her cardiomyopathy.

\section{Conclusion}

In conclusion, hyponatremia is a rare cause of Takotsubo car-

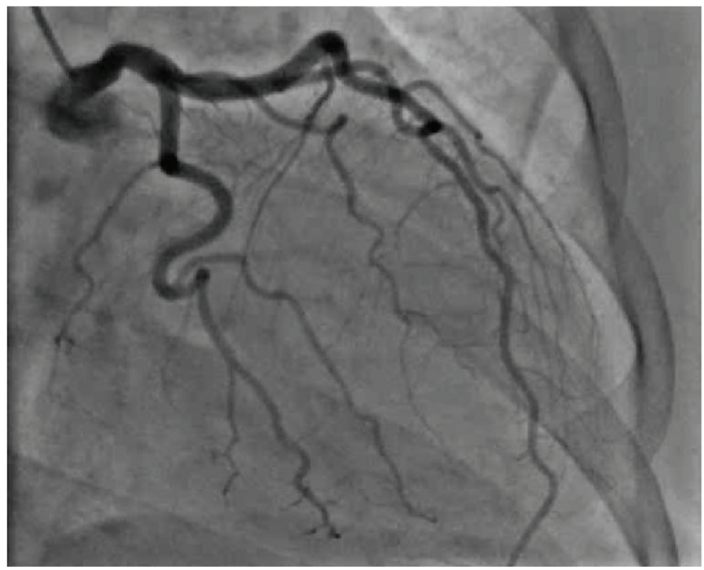

Figure 3. Coronary angiogram shortly after the episode of chest pain showing no significant coronary disease correlating with EKG findings. 
diomyopathy. Although previously reported in association with SIADH and diuretics, primary polydipsia as a cause of hyponatremia-induced Takotsubo cardiomyopathy has not been previously reported after extensive research. Physicians are encouraged to entertain a broad differential when stressinduced cardiomyopathy is diagnosed with no obvious etiology, as a broken heart may be a rare result of hyponatremia.

\section{Conflict of Interest}

The authors declare that they have no conflict of interest.

\section{References}

1. Kurowski V, Kaiser A, von Hof K, Killermann DP, Mayer B, Hartmann F, Schunkert H, et al. Apical and midventricular transient left ventricular dysfunction syndrome (tako-tsubo cardiomyopathy): frequency, mechanisms, and prognosis. Chest. 2007;132(3):809-816.

2. Siddique $M$, et al. TakoTsubo registry. Takotsubo International Registry. University Hospital Zurich. 2011.

3. Mann DL, et al. Braunwald's heart disease: a textbook of cardiovascular medicine. Tenth edition. Philadelphia, PA: Elsevier/Saunders; 2015.

4. Templin $\mathrm{C}$, et al. Clinical features and outcomes of takotsubo (stress) cardiomyopathy. The New England Journal of Medicine. U.S. National Library of Medicine. 2015.

5. Merchant EE, Johnson SW, Nguyen P, Kang C, Mallon WK. Takotsubo cardiomyopathy: a case series and review of the literature. Western Journal of Emergency Medicine. 2008.

6. Sharkey SW, Lesser JR, Maron BJ. Cardiology Patient Page. Takotsubo (stress) cardiomyopathy. Circulation. 2011;124(18):e460-462.

7. Sakuragi S, Tokunaga N, Okawa K, Kakishita M, Ohe T. A case of takotsubo cardiomyopathy associated with epileptic seizure: reversible left ventricular wall motion abnormality and ST-segment elevation. Heart Vessels. 2007;22(1):59-63.

8. Kawano H, Matsumoto Y, Arakawa S, Hayano M, Fijisawa H. Takotsubo cardiomyopathy in a patient with severe hyponatremia associated with syndrome of inappropriate antidiuretic hormone. Intern Med. 2011;50(7):727-732.

9. Daroff RB, Hitchen M, Chovan J. Bradley's neurology in clinical practice. Seventh edition. London, England: Elsevier, 2016. 